\title{
Switched Diversity Receivers over Correlated Weibull Fading Channels
}

\author{
Petros S. Bithas*, P. Takis Mathiopoulos*, and George K. Karagiannidis ${ }^{\dagger}$ \\ * Institute for Space Applications and Remote Sensing, National Observatory of Athens, \\ Metaxa \& Vas. Pavlou Street, 15236 Athens, Greece E-mail: \{pbithas;mathio\}@space.noa.gr \\ ${ }^{\dagger}$ Division of Telecommunications, Electrical and Computer Engineering Department, \\ Aristotle University of Thessaloniki, 54124 Thessaloniki, Greece E-mail: geokarag@auth.gr
}

\begin{abstract}
The problem of analyzing the performance of switch and stay combing (SSC) diversity receivers, operating over correlated and not necessarily identical distributed Weibull fading channels is considered in this paper. By means of a convenient expression for the bivariate Weibull distribution, the probability density function (PDF) of the SSC output signal-to-noise ratio (SNR) is derived in terms of the first order Marcum's Q-function. The moments of the output SNR and the corresponding cumulative distribution function (CDF) are also obtained in closed form and are utilized to derive expressions for the average output SNR, amount of fading and outage probability. By using a rapidly convergent infinite series representation for the bivariate Weibull distribution, an analytical expression for the moments generating function (MGF) is derived. The MGF can be efficiently used to evaluate the average bit error probability (ABEP) for several modulation schemes. The proposed mathematical analysis is complimented by various numerical evaluated results, which evaluate the effects of fading severity and correlation on the overall system performance.
\end{abstract}

\section{INTRODUCTION}

Diversity is a powerful communication receiver technique that offers great potential for radio link performance improvements at relatively low cost [1], [2]. There are several diversity reception methods employed in digital communication receivers including maximal-ratio combining (MRC), selection combining (SC) and switch and stay combing (SSC) [2]. Among them SSC diversity is the least complex to implement and can be used in conjunction with coherent, non-coherent and differentially coherent modulation schemes. Additionally when diversity reception techniques are employed without sufficiently separated antennas due to space limitations, e.g., small size mobile terminals, the maximum theoretical gain can not be achieved due to fading correlation [2]. As for the Weibull distribution, it fits very well to experimental fading channel measurements [3], [4] but only recently it has gained some interest [5]. It could also be considered as an alternative and generic channel model for the land-mobile satellite environment [6].

In the past there have been several papers studying the switch diversity receivers operating over correlated fading channels including [7]-[10], and more recently [11]. In [7] the performance of dual SSC receivers operating over independent and correlated Ricean fading channels is analyzed and the average bit error probability (ABEP) for non-coherent frequency shift keying (NCFSK) is derived. In [8] the impact of fading correlation of dual SSC is studied only for the case of Nakagami- $m$ fading channel. In [9] the moments output signal-to-noise ratio (SNR) and the cumulative distribution function (CDF) of SSC operating over lognormal fading channels were derived. In the same paper the average SNR and the outage probability $\left(P_{\text {out }}\right)$ have been obtained. In [10] integral representations for the MGF of SSC receiver were presented for the general case of correlated fading and nonidentical branches for several fading channels. In a recent paper [11], closed form expressions for the capacity of dual-branch MRC, SC and SSC diversity receivers over Nakagami- $m$ fading channels were derived.

For the Weibull non-correlated fading channel, some preliminary results have been presented for the error rate performance of SSC diversity receivers [12]. In [12] the channel has been considered to be uncorrelated. However, despite the practical interest for a detailed analysis of SSC receivers operating over correlated and not identical distributed (ind) Weibull fading channels, this problem has not been addressed yet in the open technical literature. Reasons for this include the absence of an exact expression of the bivariate Weibull distribution until very recently [3] and after the bivariate Weibull distribution was presented, its complicated form.

The remainder of this paper is as follows. After this introduction in Section II the system and channel model is presented. In Section III important statistical metrics and performance criteria, such as $P_{\text {out }}, \mathrm{ABEP}$, amount of 
fading (AoF) and average output SNR (ASNR) are studied. Finally in Section IV several numerical performance evaluation results are presented.

\section{System And Channel Model}

Let us consider a dual-branch diversity receiver operating over a correlated Weibull fading channel. The baseband received signal in the $\ell$ th $(\ell=1,2)$ antenna is $z_{\ell}=s h_{\ell}+n_{\ell}$, where $s$ is the transmitted complex symbol of energy $E_{s}=E\left\langle|s|^{2}\right\rangle$, with $E\langle\cdot\rangle$ denoting expectation and $|\cdot|$ absolute value, $n_{\ell}$ is the complex additive white Gaussian noise (AWGN), with single sided power spectral density $N_{0}$, and $h_{\ell}$ is the channel complex gain. The $n_{\ell}$ 's are assumed to be uncorrelated and by considering slowly varying fading the $h_{\ell}$ 's are assumed to be known at the receiver [2]. The fading envelopes $R_{1}=\left|h_{1}\right|$ and $R_{2}=\left|h_{2}\right|$ are modeled as correlated Weibull random variables. The instantaneous SNR per symbol is $\gamma_{\ell}=R_{\ell}^{2} E_{s} / N_{0}$ and the corresponding average input $\operatorname{SNR} \bar{\gamma}_{\ell}=E\left\langle R_{\ell}^{2}\right\rangle E_{s} / N_{0}=$ $\Gamma\left(1+2 / \beta_{\ell}\right) \Omega_{\ell}^{2 / \beta_{\ell}} E_{s} / N_{0}$, with $\Omega_{\ell}=E\left\langle R_{\ell}^{\beta_{\ell}}\right\rangle$ and $\Gamma(\cdot)$ being the Gamma function [13, eq. (8.310/1)].

Let $\gamma_{s s c}$ represent the instantaneous SNR per symbol at the output of the SSC and $\gamma_{\tau}$ the predetermined switching threshold. The PDF of $\gamma_{s s c}$ is given by [8]

$f_{\gamma_{s s c}}(\gamma)=\left\{\begin{array}{l}g_{s s c}(\gamma), \\ g_{s s c}(\gamma)+f_{\gamma_{1}}(\gamma),\end{array}\right.$

where

$$
\begin{aligned}
f_{\gamma_{1}}(\gamma) & =\frac{\beta_{1}}{2}\left[\frac{\Gamma\left(1+2 / \beta_{1}\right)}{\bar{\gamma}_{1}}\right]^{\beta_{1} / 2} \\
& \times \gamma^{\beta_{1} / 2-1} \exp \left[-\left(\frac{\gamma}{\bar{\gamma}_{1}} \Gamma\left(1+2 / \beta_{1}\right)\right)^{\beta_{1} / 2}\right]
\end{aligned}
$$

with $\beta_{\ell}$ expressing the severity of the fading of the $\ell$ th branch. Moreover, using [8, eq. (70)], $g_{s s c}(\gamma)$ can be expressed as

$$
g_{s s c}(\gamma)=\int_{0}^{\infty} f_{\gamma_{1} \gamma_{2}}\left(\gamma, \gamma_{2}\right) d \gamma_{2}-\int_{\gamma_{\tau}}^{\infty} f_{\gamma_{1} \gamma_{2}}\left(\gamma, \gamma_{2}\right) d \gamma_{2}
$$

where $f_{\gamma_{1} \gamma_{2}}(\cdot, \cdot)$ is the joint PDF of $\gamma_{1}, \gamma_{2}$, which can be derived by using [3, eq. (11), (30), (31)]. In this paper $g_{s s c}(\gamma)$ has been obtained in closed form in terms of the first order Marcum Q-function, [2, eq. (4.33)], as

$$
\begin{aligned}
g_{s s c}(\gamma)=\mathcal{A} \gamma^{\beta_{1} / 2-1} & \exp \left(-\mathcal{B} \gamma^{\beta_{1} / 2}\right) \\
\times & {\left[1-Q_{1}\left(\mathcal{C}_{1} \gamma^{\beta_{1} / 4}, \mathcal{D}_{2}\right)\right] }
\end{aligned}
$$

where

$$
\begin{gathered}
\mathcal{A}=\frac{\beta_{1} \beta_{2} \Gamma\left(1+2 / \beta_{1}\right)^{\beta_{1} / 2} \Gamma\left(1+2 / \beta_{2}\right)^{2} 2^{4 / \beta_{2}}}{\bar{\gamma}_{1}^{\beta_{1} / 2} \bar{\gamma}_{2}^{2} 8(1-\rho)^{4 / \beta_{2}}}, \\
\mathcal{B}=\frac{\Gamma\left(1+2 / \beta_{1}\right)^{\beta_{1} / 2}}{\bar{\gamma}_{1}^{\beta_{1} / 2}} \frac{1}{1+\sqrt{\rho}}, \\
\mathcal{C}_{\ell}=\sqrt{\frac{2 \rho}{1-\rho}} \frac{\Gamma\left(1+2 / \beta_{\ell}\right)^{\beta_{\ell} / 4}}{\bar{\gamma}_{\ell}^{\beta_{\ell} / 4}}, \\
\mathcal{D}_{\ell}=\frac{\sqrt{2} \Gamma\left(1+2 / \beta_{\ell}\right)^{\beta_{\ell} / 4}}{\sqrt{1-\rho} \bar{\gamma}_{\ell}^{\beta_{\ell} / 4}}
\end{gathered}
$$

with $\rho$ representing the Weibull correlation coefficient given in [3, eq. (14)]. Hence, by substituting (3) in (1) the PDF of $\gamma_{s s c}$ is obtained in closed-form.

\section{Statistical Metrics AND Performance ANALYSIS}

In this section using the previously derived expression for the PDF of $\gamma_{s s c}$, the outage probability $P_{\text {out }}$ and the moments will be obtained in closed form. Moreover, by using an infinite series representation for the PDF of $\gamma_{s s c}$ the MGF will be derived and consequently the ABEP will be studied.

\section{A. Outage Probability $\left(P_{\text {out }}\right)$}

Using [14, eq. (20)] the CDF of $\gamma_{s s c}$ can be obtained as

$$
\begin{aligned}
F_{\gamma_{s s c}}(\gamma)= & \operatorname{Pr}\left(\gamma_{\tau} \leq \gamma_{1} \leq \gamma\right) \\
& +\operatorname{Pr}\left(\gamma_{2}<\gamma_{\tau} \text { and } \gamma_{1}<\gamma\right)
\end{aligned}
$$

which after some manipulations can be expressed in terms of the CDF's as

$F_{\gamma_{s s c}}(\gamma)= \begin{cases}F_{\gamma_{1}, \gamma_{2}}\left(\gamma, \gamma_{\tau}\right), & \gamma \leq \gamma_{\tau} \\ F_{\gamma_{1}}(\gamma)-F_{\gamma_{2}}\left(\gamma_{\tau}\right)+F_{\gamma_{1}, \gamma_{2}}\left(\gamma, \gamma_{\tau}\right), & \gamma>\gamma_{\tau}\end{cases}$

where

$$
F_{\gamma_{\ell}}(\gamma)=1-\exp \left\{-\left[\left(\gamma / \overline{\gamma_{\ell}}\right) \Gamma\left(1+2 / \beta_{\ell}\right)\right]^{\beta_{\ell} / 2}\right\} .
$$

Moreover $F_{\gamma_{1}, \gamma_{2}}(x, y)$ is the CDF of $\gamma_{1}, \gamma_{2}$ and can be derived by using $[3$, eq. (II-2)] as

$$
\begin{array}{r}
F_{\gamma_{1}, \gamma_{2}}(x, y)=1-\exp \left[-x^{\beta_{1} / 2} \frac{\Gamma\left(1+2 / \beta_{1}\right)^{\beta_{1} / 2}}{\bar{\gamma}_{1}^{\beta_{1} / 2}}\right] \\
\times Q_{1}\left(y^{\beta_{2} / 4} \mathcal{D}_{2}, x^{\beta_{1} / 4} \mathcal{C}_{1}\right) \\
-\exp \left[-y^{\beta_{2} / 2} \frac{\Gamma\left(1+2 / \beta_{2}\right)^{\beta_{2} / 2}}{\bar{\gamma}_{2}^{\beta_{2} / 2}}\right] \\
\times\left[1-Q_{1}\left(y^{\beta_{2} / 4} \mathcal{C}_{2}, x^{\beta_{1} / 4} \mathcal{D}_{1}\right)\right] .
\end{array}
$$


Since $P_{\text {out }}$ is defined as the probability that the output SNR falls below a given threshold, $\gamma_{t h}$, it can be easily obtained for $\gamma_{s s c}$ as

$$
P_{\text {out }}\left(\gamma_{t h}\right)=F_{\gamma_{s s c}}\left(\gamma_{t h}\right) \text {. }
$$

\section{B. Moments}

By using (1) in the definition of the $n$th order moment of $\gamma_{s s c}, \mu_{\gamma_{s s c}}(n)$ [15, eq. (5.38)], yields

$$
\begin{aligned}
\mu_{\gamma_{s s c}}(n) & =\int_{0}^{\infty} \gamma^{n} g_{s s c}(\gamma) d \gamma+\int_{\gamma_{\tau}}^{\infty} \gamma^{n} f_{\gamma_{1}}(\gamma) d \gamma \\
& =\mathcal{I}_{1}+\mathcal{I}_{2} .
\end{aligned}
$$

The solution of the first integral is given in closed-form in the Appendix I. By setting $\psi=\gamma^{\beta_{1}}$ and using [13, eq. (3.381/3)], $\mathcal{I}_{2}$ yields

$$
\begin{aligned}
\mathcal{I}_{2}= & \frac{\bar{\gamma}_{1}^{n}}{\Gamma\left(1+2 / \beta_{1}\right)^{n}} \\
& \times \Gamma\left(2 n / \beta_{1}+1, \frac{\Gamma\left(1+2 / \beta_{1}\right)^{\beta_{1} / 2}}{\bar{\gamma}^{\beta_{1} / 2}}\right) \gamma_{\tau}^{\beta_{1} / 2} .
\end{aligned}
$$

where $\Gamma(\alpha, x)$ is the incomplete gamma function [13, eq. $(8.350 / 2)]$.

The ASNR, $\bar{\gamma}_{\text {out }}$, is a useful performance measure serving as an excellent indicator for the overall system's fidelity. The AoF, defined as $\operatorname{AoF} \triangleq \operatorname{var}\left(\gamma_{\mathrm{ssc}}\right) / \bar{\gamma}_{\mathrm{ssc}}^{2}$, is a unified measure of the severity of the fading channel [2], which can be expressed in terms of first- and secondorder moments of $\gamma_{s s c}$ as

$$
\mathrm{AoF}=\frac{\mu_{\gamma_{\mathrm{ssc}}}(2)}{\mu_{\gamma_{\mathrm{ssc}}}(1)^{2}}-1
$$

Closed-form expressions for both ASNR and AoF can be obtained by using (9), (A-3) and (A-2).

\section{Moments Generating Function (MGF)}

Using the (1) and (3) in the definition of the MGF, [15, eq. (5.62)], is very difficult, if not impossible, to derive the MGF of $\gamma_{s s c}, \mathcal{M}_{\gamma_{s s c}}$ in closed form. On the contrary, by using the infinite series representation for the bivariate Weibull PDF [3, eq. (19)] and after some straight forward mathematical manipulations, (2) can be expressed as

$$
g_{s s c}(\gamma)=\mathcal{E} \gamma^{\psi_{1}-1} \exp \left(-\mathcal{G}_{1} \gamma^{\beta_{1} / 2}\right)
$$

where

$$
\begin{aligned}
\mathcal{E}=\sum_{k=0}^{\infty} & \frac{\beta_{1} \beta_{2}}{4(k !)^{2}} \frac{\rho^{k}}{(1-\rho)^{2 k+1}} \frac{\Gamma\left(1+2 / \beta_{1}\right)^{\psi_{1}}}{\bar{\gamma}_{1}^{\psi_{1}}} \\
& \times \frac{\Gamma\left(1+2 / \beta_{2}\right)^{\psi_{2}}}{\bar{\gamma}_{2}^{\psi_{2}}} \frac{2 \gamma\left(k+1, \mathcal{G}_{2} \sqrt{\gamma_{\tau}}\right)}{\beta_{2} \mathcal{G}_{2}^{k+1}},
\end{aligned}
$$

$$
\mathcal{G}_{\ell}=\frac{\Gamma\left(1+2 / \beta_{\ell}\right)^{\beta_{\ell} / 2}}{(1-\rho) \bar{\gamma}_{\ell}^{\beta_{\ell} / 2}}, \psi_{\ell}=\beta_{\ell}(k+1) / 2,
$$

where $\gamma(\cdot, \cdot)$ is the lower incomplete Gamma function [13, eq. (8.350)]. Hence, using (11) $\mathcal{M}_{\gamma_{s c}}$ can be expressed as

$$
\begin{aligned}
& \mathcal{M}_{\gamma_{s s c}}(s)=\mathcal{E} \int_{0}^{\infty} \gamma^{\psi_{1}-1} \exp (-s \gamma) \\
& \times \exp \left(\mathcal{G}_{1} \gamma^{\beta_{1} / 2}\right) d \gamma+\int_{\gamma_{\tau}}^{\infty} \exp (-s \gamma) f_{\gamma_{1}}(\gamma) d \gamma \\
&=\mathcal{I}_{3}+\mathcal{I}_{4} .
\end{aligned}
$$

The same kind of integrals as $\mathcal{I}_{3}$ have been already solved in [3, eq. (8)] as

$$
\begin{gathered}
\mathcal{I}_{3}=\frac{\mathcal{E}}{s^{\psi_{1}}} \frac{\lambda^{\psi_{1}} \sqrt{\kappa / \lambda}}{(\sqrt{2 \pi})^{\kappa+\lambda-2}} \\
G_{\lambda, \kappa}^{\kappa, \lambda}\left[\left(\frac{\mathcal{G}_{1}}{s^{\beta_{1} / 2}}\right)^{\kappa} \frac{\lambda^{\lambda}}{\kappa^{\kappa}} \mid \begin{array}{c}
\left(1-\psi_{1}\right) / \lambda,\left(2-\psi_{1}\right) / \lambda, \cdots,\left(\lambda-\psi_{1}\right) / \lambda \\
0,
\end{array}\right],
\end{gathered}
$$

where $\lambda / \kappa=\beta_{1} / 2$.

Moreover, by representing the exponential as infinite series [13, eq. (1.211/1)], using [13, eq. (3.381/3)] and after some straight forward mathematical manipulations $\mathcal{I}_{4}$ can be solved as

$$
\begin{aligned}
\mathcal{I}_{4}=\sum_{k=0}^{\infty} & \frac{(-s)^{k}}{k !} \frac{\bar{\gamma}_{1}}{\Gamma\left(1+2 / \beta_{1}\right)^{k}} \\
& \times \Gamma\left[2 k / \beta_{1}+1, \frac{\Gamma\left(1+2 / \beta_{1}\right)^{\beta_{1} / 2}}{\bar{\gamma}_{1}^{\beta_{1} / 2}} \gamma_{\tau}^{\beta_{1} / 2}\right] .
\end{aligned}
$$

Using the MGF-based approach [2] the ABEP of binary differential phase shift keying (BDPSK) can be derived directly by setting $s=1$ in (13) and (14).

\section{Numerical Results}

Based on the proposed formulation, representative performance criteria of SSC diversity receivers have been numerically evaluated, including the ABEP and $P_{\text {out }}$. In Fig. 1, the ABEP performance of dual branch SSC is illustrated for BDPSK signaling as a function of the average input SNR per bit, $\bar{\gamma}_{b}=\bar{\gamma} / \log _{2} M$, and for several values of $\beta^{1}$. As expected, the ABEP improves as $\bar{\gamma}$ increases, while for a fixed value of $\bar{\gamma}$, ABEP improves as $\beta$ and/or $\rho$ decreases. Moreover, the curves

\footnotetext{
${ }^{1}$ For the convenience of the presentation of the performance results, it is assumed that $\beta_{1}=\beta_{2}=\beta$ and $\bar{\gamma}_{1}=\bar{\gamma}_{2}=\bar{\gamma}$.
} 


$$
\begin{array}{r}
\mathcal{I}_{1}^{B}=\frac{1}{2}\left(\frac{2 n}{\beta_{1}}\right) ! \frac{\bar{\gamma}_{1}^{n+\beta_{1} / 2}(1-\rho)^{2 n / \beta_{1}+1}}{\Gamma\left(1+2 / \beta_{1}\right)^{n+\beta_{1} / 2}(1-\sqrt{\rho})^{2 n / \beta_{1}+1}} \frac{\rho}{\rho-\sqrt{\rho}+1} \exp \left[-\frac{\Gamma\left(1+2 / \beta_{2}\right)^{\beta_{2} / 2} \gamma_{\tau}^{\beta_{2} / 2}(1-\sqrt{\rho})}{\bar{\gamma}_{2}^{\beta_{2} / 2}(\rho-\sqrt{\rho}+1)(1-\rho)}\right] \\
\quad \times \sum_{\nu=0}^{2 n / \beta_{1}} \epsilon_{\nu}\left(\frac{1-\sqrt{\rho}}{\rho-\sqrt{\rho}+1}\right)^{\nu} L_{\nu}\left[-\frac{\Gamma\left(1+2 / \beta_{2}\right)^{\beta_{2} / 2} \rho \gamma_{\tau}^{\beta_{2} / 2}}{\bar{\gamma}_{2}^{\beta_{2} / 2}(1-\rho)(\rho-\sqrt{\rho}+1)}\right] .
\end{array}
$$

also indicate that the differences among them become less significant as either $\beta$ increases and/or $\rho$ decreases.

In Fig. 2, $P_{\text {out }}$ is plotted as a function of the normalized outage threshold $\gamma_{t h} / \bar{\gamma}_{b}$ for several values of $\beta$ and $\rho$. The exponentially power decaying profile (PDP) has been considered [16], [17], i.e., $\bar{\gamma}_{\ell}=\bar{\gamma}_{1} \exp [-\delta(\ell-1)]$, where $\delta$ is the power decaying factor. In Fig. 2 it is illustrated that as $\beta$ increases and/or $\rho$ decreases $P_{\text {out }}$ decreases.

\section{APPENDIX I}

\section{DERIVATION OF INTEGRAL $\mathcal{I}_{1}$ IN CLOSED-FORM}

Using (2), $\mathcal{I}_{1}$ can be expressed as

$$
\begin{gathered}
\mathcal{I}_{1}=\mathcal{A}\left[\int_{0}^{\infty} \gamma^{n+\beta_{1} / 2-1} \exp \left(-\mathcal{B} \gamma^{\beta_{1} / 2}\right) d \gamma\right. \\
\left.-\int_{0}^{\infty} \gamma^{n+\beta_{1} / 2-1} \exp \left(-\mathcal{B} \gamma^{\beta_{1} / 2}\right) Q_{1}\left(\mathcal{C} \gamma^{\beta_{1} / 4}, \mathcal{D}\right) d \gamma\right] \\
\quad=\mathcal{A}\left(\mathcal{I}_{1}^{A}-\mathcal{I}_{1}^{B}\right) .
\end{gathered}
$$

By setting $x=\gamma^{\beta_{1} / 2}$ and using [13, eq. (3.381/4)], $\mathcal{I}_{1}^{A}$ can be solved as

$$
\mathcal{I}_{1}^{A}=\frac{2 \Gamma\left(2 n / \beta_{1}+1\right)}{\mathcal{B}^{2 n / \beta_{1}+1} \beta_{1}} .
$$

Moreover, by setting $x=\gamma^{\beta_{1} / 4}$, using [18, eq. (9)] and after some mathematical simplifications $\mathcal{I}_{1}^{B}$ can be solved as in (A-3)(top of this page). In (A-3) $L_{\nu}(\cdot)$ is the Laguerre polynomials [13, eq. (8.970/1)] and $\epsilon_{\nu}$ is given by

$\epsilon_{\kappa}= \begin{cases}1, & \nu<\frac{2 n}{\beta_{1}} \\ 1+\frac{\left[\Gamma\left(1+2 / \beta_{1}\right)^{\beta_{1} / 2}\right] /\left[\bar{\gamma}_{1}^{\beta_{1} / 2}(1-\sqrt{\rho}) /(1-\rho)\right]}{\left[\rho \Gamma\left(1+2 / \beta_{1}\right)^{\beta_{1} / 2}\right] /\left[(1-\rho) \bar{\gamma}_{1}^{\beta_{1} / 2}\right]}, & \nu=\frac{2 n}{\beta_{1}} .\end{cases}$

\section{ACKNOWLEDGMENTS}

This work has been performed within the framework of the Satellite Network of Excellence (SatNEx-II) project (IST-027393), a Network of Excellence (NoE) funded by European Commission (EC) under the FP6 program.

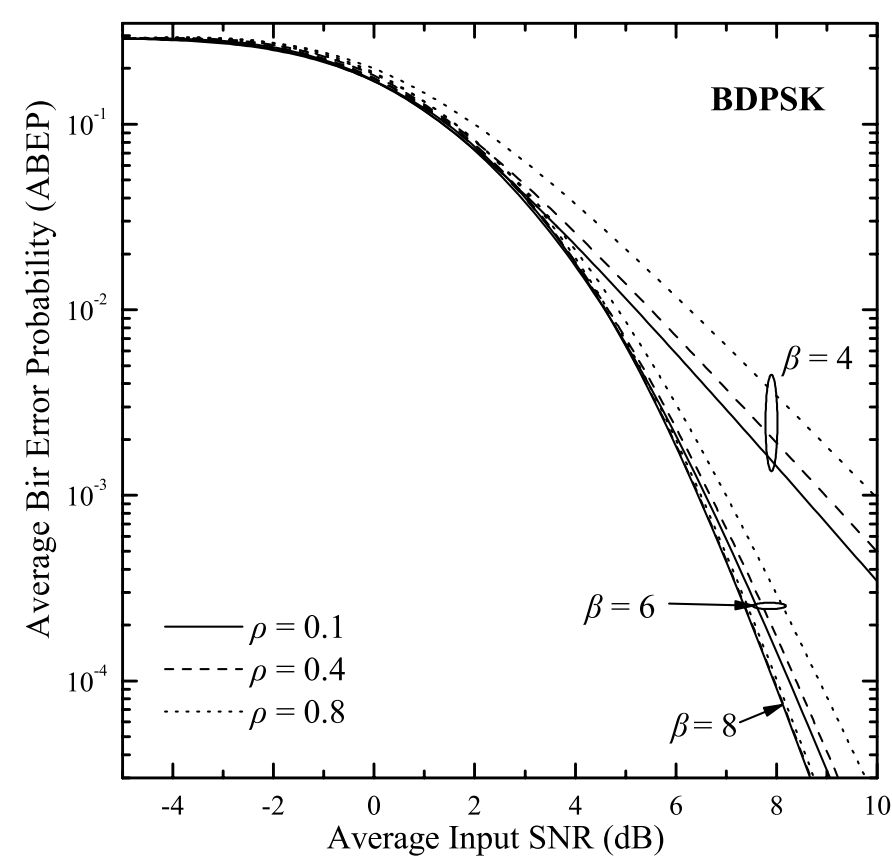

Fig. 1. ABEP of BDPSK signaling versus $\bar{\gamma}$ in a correlated Weibull fading environment.

\section{REFERENCES}

[1] T. S. Rappaport, Wireless Communications. New Jersey: Prentice Hall PTR, 1996.

[2] M. K. Simon and M.-S. Alouini, Digital Communication over Fading Channels, 2nd ed. New York: Wiley, 2005.

[3] N. C. Sagias and G. K. Karagiannidis, "Gaussian class multivariate Weibull distributions: Theory and applications in fading channels," IEEE Trans. Inform. Theory, vol. 51, no. 10, pp. 3608-3619, Oct. 2005.

[4] F. Babich and G. Lombardi, "Statistical analysis and characterizasion of the indoor propagation channel," IEEE Trans. Commun., vol. 48, pp. 455-464, Mar. 2000.

[5] N. C. Sagias, "Capacity of dual-branch selection diversity receivers in correlative Weibull fading," Wiley European Transactions on Telecommunications, vol. 17, no. 1, pp. 37-43, Jan/Feb 2006.

[6] P. S. Bithas, G. K. Karagiannidis, N. C. Sagias, P. T. Mathiopoulos, S. A. Kotsopoulos, and G. Corazza, "Performance analysis of a class of GSC receivers over nonidentical Weibull fading channels," IEEE Trans. Veh. Technol., vol. 54, no. 6, pp. 1963-1970, Nov. 2005. 


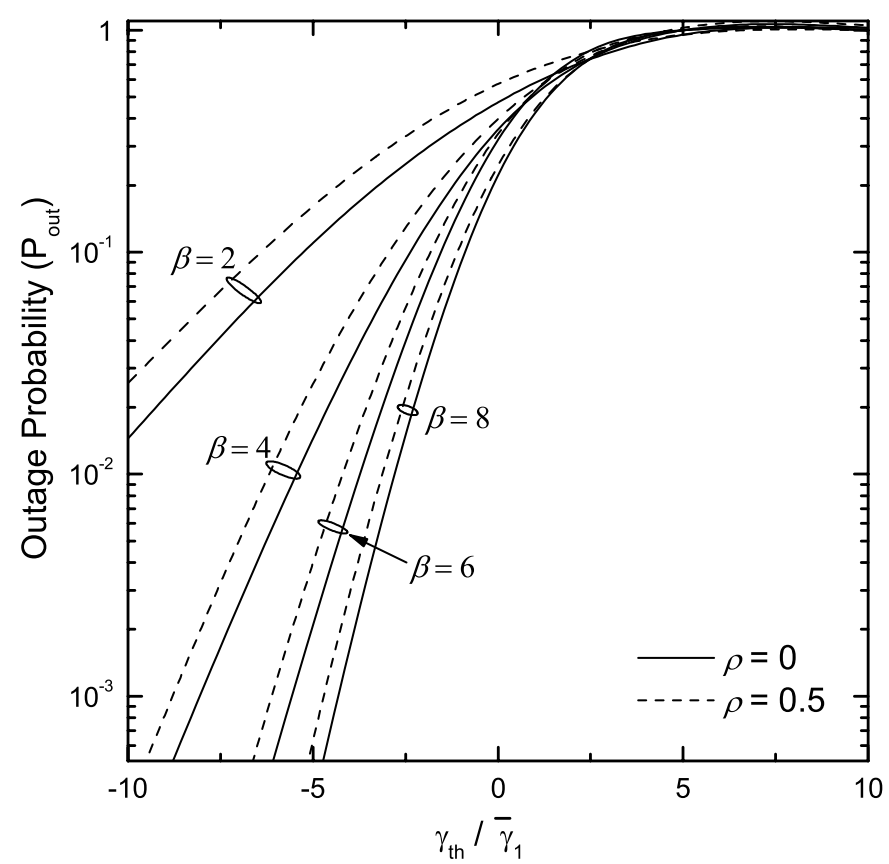

Fig. 2. Outage probability $\left(P_{\text {out }}\right)$ versus the normalized outage threshold for several values of $\beta$ and $\rho$.

[7] A. A. Abu-Dayya and N. C. Beaulieu, "Switched diversity on microcellular Ricean channels," IEEE Trans. Veh. Technol., vol. 43, no. 4, pp. 970-976, Nov. 1994.

[8] Y. C. Ko, M.-S. Alouini, and M. K. Simon, "Analysis and optimization of switched diversity systems," IEEE Trans. Veh. Technol., vol. 49, no. 5, pp. 1813-1831, Sept. 2000.
[9] M.-S. Alouini and M. K. Simon, "Dual diversity over correlated log-normal fading channels," IEEE Trans. Commun., vol. 50, no. 12, pp. 1946-1959, Dec. 2002.

[10] C. Tellambura, A. Annamalai, and V. K. Bhargava, "Unified analysis of switched diversity systems in independent and correlated fading channels," IEEE Trans. Commun., vol. 49, no. 11, pp. 1955-1965, Nov. 2001.

[11] S. Khatalin and J. P. Fonseka, "Capacity of correlated Nakagami- $m$ fading channels with diversity combining techniques," IEEE Trans. Veh. Commun., vol. 55, no. 1, pp. 142150, Jan. 2006.

[12] N. C. Sagias, G. K. Karagiannidis, and G. S. Tombras, "Errorrates analysis of switched diversity receivers in Weibull fading," Electron. Letters, vol. 40, no. 11, May 2004.

[13] I. S. Gradshteyn and I. M. Ryzhik, Table of Integrals, Series, and Products, 6th ed. New York: Academic Press, 2000.

[14] A. A. Abu-Dayya and N. C. Beaulieu, "Analysis of switched diversity systems on generalized-fading channels," IEEE Trans. Commun., vol. 42, no. 11, pp. 2959-2964, Nov. 1994.

[15] A. Papoulis, Probability, Random Variables, and Stochastic processes, 2nd ed. McGraw-Hill, 1984.

[16] Y. Ma and S. Pasupathy, "Efficient performance evaluation for generalized selection combining on generalized fading channels," IEEE Trans. Wireless Commun., vol. 3, no. 1, pp. 29-34, Jan. 2004.

[17] N. C. Sagias, D. A. Zogas, and G. K. Karagiannidis, "Selection diversity receivers over nonidentical Weibull fading channels," IEEE Trans. Veh. Technol., vol. 54, no. 6, pp. 2146-2151, Nov. 2005.

[18] A. H. Nuttal, "Some integrals involving the $Q_{M}$ function," IEEE Trans. Inform. Theory, pp. 95-96, Jan. 1975. 